\title{
DYNAMICS AND SYMMETRIES \\ OF A FIELD PARTITIONED BY \\ AN ACCELERATED FRAME
}

\author{
Ulrich H. Gerlach \\ Department of Mathematics \\ The Ohio State University \\ Columbus, Ohio 43210
}

\begin{abstract}
The canonical evolution and symmetry generators are exhibited for a Klein-Gordon (K-G) system which has been partitioned by an accelerated coordinate frame into a pair of subsystems. This partitioning of the $\mathrm{K}-\mathrm{G}$ system is conveyed to the canonical generators by the eigenfunction property of the Minkowski Bessel (M-B) modes. In terms of the M-B degrees of freedom, which are unitarily related to those of the Minkowski plane waves, a near complete diagonalization of these generators can be realized.
\end{abstract}

\section{TABLE OF CONTENTS}

I. MOTIVATIONS

II. SYMMETRY GENRATORS FOR THE PARTITIONED SYSTEM

A. Subsystem Generators: Partial Diagonalization

1. The Hamiltonian $H_{I}$

2. Minkowski Bessel Modes

3. The Hamiltonian $H_{I}$ (continued)

4. The Transverse Subsystem Momenta $\vec{P}_{I}$ and $\vec{P}_{I I}$. B. Total System Generators: Complete Diagonalization

III. INERTIAL TRANSLATION GENERATORS FOR THE TOTAL SYSTEM

IV. CONCLUSION

\section{MOTIVATIONS}

Both within a classical and a quantum mechanical framework all inertial frames are equivalent. This is expressed by the fact that the groundstates of a relativistic wave field in 
these frames are one and the same. Thus all inertial refrigerators cooling the wave field produce the same quantum state, the Minkowski vacuum.

1. Considers however an accelerated refrigerator. More specifically consider a pair of refrigerators accelerating linearly and uniformly into opposite directions. Their cooling operation results a quantum state that is strikingly different from the Minkowski vacuum. Its macroscopic manifestation can be characterized as a superfluid condensate of liquid light ${ }^{1,2,3}$.

These refrigerators are each situated in their own respective linearly uniformly accelerated coordinate frames. The acceleration is such that these two frames are causally disjoint but jointly contain a Cauchy surface for all of Minskowski space-time. Consequently these two frames partition the wave field quantum system into two subsystems, which are respectively cooled by each of the two refrigerators. Each accelerated frame is static. The time evolution of the wave field in a frame is governed by its Hamiltonian. Any one of the field's stationary states is characterized by its conserved "boost" energy. The ground state for the subsystem is the Fulling state ${ }^{4}$. There also is a Fulling state for the other subsystem. The product of these two respective Fulling states is a quantum state for the total wave field system. In the framework of condensed matter physics this total wave field state is called a condensed vacuum (= "Rindler" 5,6 ) state. The motivation for this name is that on a macroscopic scale this state manifests itself as liquid light, a photon superfluid condensate ${ }^{1}$.

The framework within which condensed matter physics considerations arise is a KleinGordon system that has been partitioned by two accelerated frames into two subsystems whose symmetries and evolution bears a nonstandard relation to those of the total K-G system.

2. One can obtain a general idea of the unusual relation between the pair of subsystems and the total system by contrasting the partioned K-G system, which has infinitely many quantized degrees of freedom, with an atomic system consisting of a pair of coupled angular momenta, which has only finitely many degrees of freedom. For the finite atomic system the familiar Clebsch-Gordon technology establishes a unitary relation between the pair of subsystems and the total systems. Guided by group representation theory, this technology allows one to classify and construct very systematically irreducible spaces of quantum states of the total system from the product of those of each subsystem. For the atomic system the symmetry group is the rotation group. It is unitarily implemented on the product states of the pair of subsystems as well as on those of the total system.

By contrast the symmetry group for the partitioned Klein-Gordon system is the two dimensional Poincare group (= "semidirect product" of translations and boosts on 2-D Lorentz space time ${ }^{7}$ ). It can be implemented on the dynamical variables of the total system. It can not however be unitarily implemented on the quantum states of the system. In particular translations in the Lorentz plane do not have a unitary representation: the condensed vacuum state (= product of two Fulling vacua) is not translation invariant; it "breaks" the translational symmetry subgroup ${ }^{3}$. A translation would produce what amounts to an excited state with an infinite number of quasiparticles. It follows that this 
breaking of translational symmetry by the condensed vacuum state is an obstruction to constructing and classifying the K-G quantum states into irreducible unitary representation spaces of the two dimensional Poincare group in the Lorentz plane.

3. To impose boundary conditions on a field, at some stage it invariably becomes necessary that one incorporate the space-time features of the reference frame. Take, for example, a Klein Gordon field in an inertial reference frame. This frame is equipped with the usual Minkowski coordinates and one therefore resolves the Klein-Gordon field into the familiar degrees of freedom, the Minkowski plane wave modes. They express the translation invaraince of the frame, and they also make the implementation for the Feynman boundary conditions very easy.

If, by contrast, the field is described so as to incorporate the space-time features of a pair of accelerated frames, then one resolves the field into a different set of degrees of freedom, the Minkowski Bessel ${ }^{8}$ (M-B) modes.

These M-B modes readily leads to the total K-G system being partitioned into a pair of independent subsystems corresponding to the two accelerated frames. Furthermore these modes, being Lorentz boost eigenfunctions, express the invariance of the union of the two accelerated frames under Lorentz boosts.

The incorporation of the space-time features of an accelerated frame can be extended from the field to its canonical symmetry and evolution generators. These quadratic expressions are a vital ingredient for the quantum theory of the partitioned K-G system and as such give vital information about it.

The purpose of this paper is to give for each of the two K-G subsystems the Minkowski Bessel mode expansion of their evolution and symmetry generators: they are the Lorentz boost, i.e. moment of mass energy and the generators of translation, i.e. linear momentum, into the transverse directions (Section II.A). The analogous generators are given for the total K-G system (Section II.B). Similarly for the total K-G system we give such Minkowski Bessel mode expansions for all four translation generators (Section III) 


\section{SYMMETRY GENERATORS FOR THE PARTITIONED SYSTEM}

If a wave field system is invariant under a space-time symmetry generated by a ("Killing") vector field

$$
\xi^{\mu} \frac{\partial}{\partial x^{\mu}}: \xi_{\mu ; \nu}+\xi_{\nu ; \mu}=0,
$$

then the associated conserved current satisfies

$$
\left(\xi^{\mu} T_{\mu}^{\nu}\right) ;_{\nu}=0
$$

Here $T_{\mu}^{\nu}$ is the stress-energy tensor which for a Klein-Gordon (K-G) system is

$$
T_{\mu \nu}=\psi,{ }_{\mu} \psi{ }_{\nu}-\frac{1}{2} g_{\mu \nu}\left(\psi,{ }_{\alpha} \psi, \beta g^{\alpha \beta}+m^{2} \psi^{2}\right)
$$

The conserved quantity which remains invariant under the symmetry transformation is

$$
\iint_{\substack{\text { space-like } \\ \text { hypersurface }}} \int \xi^{\mu} T_{\mu}^{\nu} d^{3} \Sigma_{\nu} .
$$

Consider the three Killing vector fields

$$
\begin{aligned}
\xi_{\tau}^{\mu} x \frac{\partial}{\partial x^{\mu}} & =\frac{\partial}{\partial t}+t \frac{\partial}{\partial x} \equiv \frac{\partial}{\partial \tau} \\
\xi_{y}^{\mu} \frac{\partial}{\partial x^{\mu}} & =\frac{\partial}{\partial y} \\
\xi_{z}^{\mu} \frac{\partial}{\partial x^{\mu}} & =\frac{\partial}{\partial z}
\end{aligned}
$$

and their corresponding canonical generators

$$
\begin{aligned}
P_{\tau} & =\iiint \xi_{\tau}^{\mu} T_{\mu}^{\nu} d^{3} \Sigma_{\nu} \\
P_{y} & =\iiint \xi_{y}^{\mu} T_{\mu}^{\nu} d^{3} \Sigma_{\nu} \\
P_{z} & =\iiint \xi_{z}^{\mu} T_{\mu}^{\nu} d^{3} \Sigma_{\nu}
\end{aligned}
$$

where the integration is over a global space-like hypersurface. 
The Rindler coordinates $\xi$ and $\tau$ that mold themselves to these Lorentz and translation vector fields, Eqs. (2.4), are related to the MInkowski coordinates by

$$
\begin{aligned}
t & = \pm \xi \sinh \tau \\
x & = \pm \xi \cosh \tau
\end{aligned}
$$

(The coordinate $\xi$ is not to be confused with the vector symbol $\xi^{\mu}$ ). Here $0<\xi<$ $\infty,-\infty<\tau<\infty$ and the two signs refer to the two respective accelerated coordinate frames I and II. In both of them the metric assumes the form

$$
d s^{2}=-\xi^{2} d t^{2}+d \xi^{2}+d y^{2}+d z^{2} .
$$

The space-like integration surface of the canonical genrators, Eqs. (2.5), is partitioned by the pair of accelerated frames into the two parts

$$
\{\tau=\text { const } ;-\infty>-\xi>0\} \text { which lies in } I I
$$

and

$$
\{\tau=\text { const } 0<\xi<\infty\} \text { which lies in } I .
$$

All integration volume components $d^{3} \Sigma_{\nu}$ are therefore zero, except

$$
d^{3} \Sigma_{\tau}=-\xi d \xi d y d z
$$

which holds both in $I I$ and in $I$.

Inserting Eqs. (2.2), (2.4), and (2.7) into Eqs. (2.5) and keeping track of the orientation of the integration hypersurface in II, one obtains

$$
\begin{aligned}
P_{\tau} & =H_{I I}+H_{I} \\
& =\left.\frac{1}{2} \int_{\infty}^{0} \int_{-\infty}^{\infty} \int_{-\infty}^{\infty}\{\cdots\}\right|_{I I} d \xi d y d z+\left.\frac{1}{2} \int_{0}^{\infty} \int_{-\infty}^{\infty} \int_{-\infty}^{\infty}\{\cdots\}\right|_{I} d \xi d y d z
\end{aligned}
$$

with

$$
\{\cdots\}=\left\{\frac{1}{\xi}\left(\psi_{,}\right)^{2}+\xi\left[(\psi, \xi)^{2}+\left(\psi_{,_{y}}\right)^{2}+(\psi, z)^{2}+m^{2} \psi^{2}\right]\right\},
$$

and

$$
\begin{aligned}
\vec{P} & =\vec{P}_{I I}+\vec{P}_{I} \\
& =\int_{\infty}^{0} \int_{-\infty}^{\infty} \int_{-\infty}^{\infty}\left\{\frac{1}{\xi} \psi,{ }_{\tau} \vec{\nabla} \psi\right\} d \xi d y d z+\int_{0}^{\infty} \int_{-\infty}^{\infty} \int_{-\infty}^{\infty}\left\{\frac{1}{\xi} \psi,{ }_{\tau} \vec{\nabla} \psi\right\} d \xi d y d z
\end{aligned}
$$


with

$$
\vec{P} \equiv\left(P_{y}, P_{z}\right), \quad \vec{\nabla} \psi \equiv\left(\frac{\partial \psi}{\partial y}, \frac{\partial \psi}{\partial z}\right)
$$

Thus the Lorentz boost generator and the transverse translation generator decompose into pats which affect the pair of K-G subsystems

with

$$
\vec{P} \equiv\left(P_{y}, P_{z}\right), \quad \vec{\nabla} \psi \equiv\left(\frac{\partial \psi}{\partial y}, \frac{\partial \psi}{\partial z}\right)
$$

The physical interpretation of the boost generator $P_{\tau}$ depends on the frame. Relative to the accelerated frame $I, H_{I}$ is the conserved energy because $\tau$ is the time parameter. Similarly $H_{I I}$ is the conserved energy for the accelerated frame $I I$. Relative to the inertial frame the interpretation is different. The Minkowski frame integral, obtained from Eqs. (2.4) and (2.5),

$$
P_{\tau}=\iiint_{t=0} x T_{00} d x d y d z
$$

implies that $P_{\tau}$ is the total moment of mass-energy of the K-G system around the event $t=x=0$. By contrast $H_{I}$ is the partial moment of mass energy of subsystem $I$.

\section{A. Substystem Generators: Partial Diagonalization}

We shall reexpress the subsystem I evolution generator

$$
\left.H_{I}=\frac{1}{2} \int_{0}^{\infty} \int_{-\infty}^{\infty} \int_{-\infty}^{\infty}\left\{\frac{1}{\xi}(\psi, \tau)^{2}+\xi[\psi, \xi)^{2}+(\psi, y)^{2}+(\psi, z)^{2}+m^{2} \psi^{2}\right]\right\} d \xi d y d(\xi 2
$$

and the two subsystem $I$ momentum components

$$
\vec{P}_{I}=\int_{0}^{\infty} \int_{-\infty}^{\infty} \int_{-\infty}^{\infty}\left\{\frac{1}{\xi} \psi,{ }_{\tau} \vec{\nabla} \psi\right\} d \xi d y d z
$$

in terms of the Minkowski Bessel modes.

1.) The Hamiltonian $\mathrm{H}_{\mathrm{I}}$

First focus on the observer Hamiltonian $H_{I}$, Eq. (2.10). The task is simplified considerably with the help of the of the K-G field equation. It can be used to eliminate reference to derivatives with respect to the non-cyclic coordinate $\xi$ :

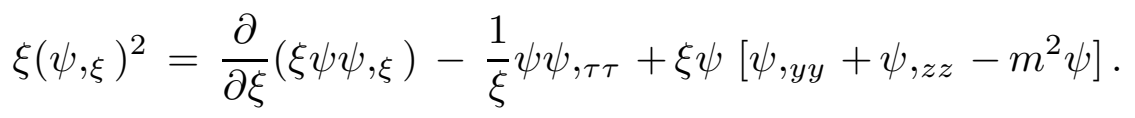


Thus the subsystem Hamiltonian $H_{I}$, reduces to

$$
\begin{aligned}
H_{I} & =\frac{1}{2} \int_{0}^{\infty} \int_{-\infty}^{\infty} \int_{-\infty}^{\infty}\left\{\left(\psi,_{\tau} \psi,_{\tau}-\psi \psi,_{\tau \tau}\right) / \xi+\left(\psi,,_{y} \psi,_{y}+\psi \psi,_{y y}\right) \xi\right. \\
& \left.+\left(\psi,_{z} \psi,_{z}+\psi \psi,_{z z}\right) \xi\right\} d \xi d y d z .
\end{aligned}
$$

Here we have performed an integration by parts (w.r.t. $\xi$ ) and dropped the surface terms. Now introduce the K-G field expanded in terms of the globally defined Minkowski-Bessel modes

$B_{\omega}^{ \pm}(k U, k V)$.

2. The Minkowski Bessel Modes

These mode are defined on two dimensional Lorentz space-time spanned by $t=\frac{1}{2}(V+$ $U$ ) and $x=\frac{1}{2}(V-U)$ and are given by ${ }^{8}$

$$
\begin{aligned}
B_{\omega}^{ \pm}(k U, k V) & =\frac{1}{2 \pi} \int_{-\infty}^{\infty} e^{\mp i\left(\omega_{k} t-k_{x} x\right)} e^{-i \omega \theta} d \theta \\
& =\frac{1}{2 \pi} \int_{-\infty}^{\infty} \int_{-\infty}^{\infty} \exp \left[\mp i k\left(U e^{\theta}+V e^{-\theta}\right) / 2\right] e^{-i \omega \theta} d \theta
\end{aligned}
$$

with

$$
\begin{aligned}
\omega_{k} & =k \cosh \theta \\
k_{x} & =k \sinh \theta \\
k & =\sqrt{k_{y}^{2}+k_{z}^{2}+m^{2}}>0
\end{aligned}
$$

Their coordinate representatives for $I$ and $I I$ are

$$
B_{\omega}^{ \pm}(k U, k V)=\frac{1}{\pi} K_{i \omega}(k \xi) e^{-i \omega \tau}\left\{\begin{array}{llc}
e^{ \pm \pi \omega / 2} & \text { in } & I \\
e^{\mp \pi \omega / 2} & \text { in } & I I
\end{array}\right.
$$

and they have the following relevant properties:

(i)

$$
\frac{\partial}{\partial \tau} B_{\omega}^{ \pm}=-i \omega B_{\omega}^{ \pm}
$$

(ii)

$$
\left(B_{\omega}^{+}\right)^{*}=B_{-\omega}^{-}
$$


(iii)

$$
\int_{0}^{\infty} B_{\omega}^{+} B_{ \pm \omega^{\prime}}^{ \pm} \frac{d \xi}{\xi}=\frac{e^{-i\left(\omega \pm \omega^{\prime}\right) \tau}}{2 \omega \sinh \pi \omega}\left[\delta\left(\omega+\omega^{\prime}\right)+\delta\left(\omega-\omega^{\prime}\right)\right]\left\{\begin{array}{ccc}
e^{\pi\left(\omega+\omega^{\prime}\right) / 2} & \text { in } & I \\
e^{-\pi\left(\omega+\omega^{\prime}\right) / 2} & \text { in } & I I
\end{array}\right\}
$$

The last integral follows from

$$
\int_{0}^{\infty} K_{i \omega}(k \xi) K_{i \omega^{\prime}}(k \xi) \frac{d \xi}{\xi}=\frac{\pi^{2}}{2 \omega \sinh \pi \omega}\left[\delta\left(\omega+\omega^{\prime}\right)+\delta\left(\omega_{-} \omega^{\prime}\right)\right]
$$

and Eq. (2.15)

3. The Hamiltonian $\mathrm{H}_{\mathrm{I}}$ (continued).

Expanded in terms of these modes the K-G field is

$$
\psi=(1 / \sqrt{2}) \int_{-\infty}^{\infty} \int_{-\infty}^{\infty} \int_{-\infty}^{\infty} a_{\omega k} B_{\omega}^{+} \frac{e^{i\left(k_{y} y+k_{z} z\right)}}{2 \pi}+a_{\omega k}^{+}\left(B_{\omega}^{+}\right)^{*} \frac{e^{-i\left(k_{y} y+k_{z} z\right)}}{2 \pi} d \omega d k_{y} d\left(\mathbb{2}_{z} 17\right)
$$

The last two integrals in Eq. (2.12) (involving the spatial derivations) vanish. This is because with $\psi(x)$ given by Eq. (2.17), the first one, say, is proportional to

$$
\int_{-\infty}^{\infty} \int_{-\infty}^{\infty} \int_{-\infty}^{\infty}\left[ \pm k_{y}^{\prime} k_{y}+k_{y}^{\prime} k_{y}^{\prime}\right] \delta\left(k_{y} \pm k_{y}^{\prime}\right) d k_{y} d k_{y}^{\prime}=0 .
$$

The second one involving $k_{z}$ and $k_{z}^{\prime}$ also vanishes. Thus the observer Hamiltonian $H_{I}$ reduces to

$$
H_{I}=\frac{1}{2} \int_{0}^{\infty} \int_{-\infty}^{\infty} \int_{-\infty}^{\infty} \frac{1}{2}\left(\psi_{\tau} \psi_{,}-\psi \psi,_{\tau \tau}\right) \xi^{-1} d \xi d y d z
$$

This Hamiltonian can be readily expressed in terms of the quantum operators $a_{\omega k}$ and $a_{\omega k}^{+}$. The field $\psi$, Eq. (2.17), is given by

$$
\psi=\psi^{+}+\psi^{-}=\psi^{+}+\text {c.c. }
$$

where

$$
\psi^{+}=(1 / \sqrt{2}) \int_{-\infty}^{\infty} \int_{-\infty}^{\infty} \int_{-\infty}^{\infty} B_{\omega}^{+}(k U, k V) a_{\omega k} \frac{e^{i k_{y} y+i k_{z} z}}{2 \pi} d \omega d k_{y} d k_{z}
$$


Thus the Hamiltonian $H_{I}$, Eq. (2.18), becomes

$$
\begin{aligned}
H_{I} & =\frac{1}{2} \int_{0}^{\infty} \int_{-\infty}^{\infty} \int_{-\infty}^{\infty}\left\{\psi_{, \tau}^{+}\left(\psi, \tau_{\tau}^{+}+\psi,_{\tau}^{-}\right)-\psi,,_{\tau}^{+}\left(\psi^{+}+\psi^{-}\right)+c . c .\right\} \xi^{-1} d \xi d y d z \\
& =\frac{1}{2} \int_{0}^{\infty} \xi^{-1} d \xi \int_{-\infty}^{\infty} \int_{-\infty}^{\infty} \int_{-\infty}^{\infty} d \omega d^{2} k \int_{-\infty}^{\infty} \int_{-\infty}^{\infty} \int_{-\infty}^{\infty} d \omega^{\prime} d^{2} k^{\prime} \\
& \frac{1}{2}\left\{(-) B_{\omega}^{+} \omega a_{\omega k} \omega^{\prime}\left[B_{\omega^{\prime}}^{+} a_{\omega^{\prime} k^{\prime}} \delta^{2}\left(k+k^{\prime}\right)+\left(B_{\omega^{\prime}}^{+}\right)^{*} a_{\omega^{\prime} k^{\prime}}^{+} \delta^{2}\left(k-k^{\prime}\right)\right]\right. \\
& \left.-(-) B_{\omega}^{+} \omega^{2} a_{\omega k}\left[B_{\omega^{\prime}}^{+} a_{\omega^{\prime} k^{\prime}} \delta^{2}\left(k+k^{\prime}\right)+\left(B_{\omega^{\prime}}^{+}\right)^{*} a_{\omega^{\prime} k^{\prime}}^{+} \delta^{2}\left(k-k^{\prime}\right)\right]+c . c .\right\}
\end{aligned}
$$

where we used the eigenfunction property, Eq. (2.16a)

The Minkowski Bessel modes $B_{\omega}^{ \pm}$are even functions of $k_{y}$ and $k_{z}$. This follows from Eqs. (2.13) and (2.14). Thus there is no difficulty in doing the integration $\iint \cdots d^{2} k^{\prime}$. The step next to the last is to perform the integration over the remaining spatial coordinate $\xi$. Using Eqs. (2.16b) together with (2.16c) one obtains the Klein-Gordon subsystem Hamiltonian $H_{I}$,

$$
\begin{aligned}
H_{I} & =\frac{1}{2} \int_{-\infty}^{\infty} \int_{-\infty}^{\infty} \int_{-\infty}^{\infty} d \omega d^{2} k \int_{-\infty}^{\infty} d \omega^{\prime} \frac{1}{2 \omega \sinh \pi \omega} \\
& \frac{1}{2}\left\{\left(-\omega \omega^{\prime}+\omega^{2}\right) e^{-i\left(\omega+\omega^{\prime}\right) \tau} e^{\pi\left(\omega+\omega^{\prime}\right) / 2}\left[\delta\left(\omega-\omega^{\prime}\right)+\delta\left(\omega+\omega^{\prime}\right)\right] a_{\omega k} a_{\omega^{\prime}-k}\right. \\
& \left.+\left(\omega \omega^{\prime}+\omega^{2}\right) e^{-i\left(\omega-\omega^{\prime}\right) \tau} e^{\pi\left(\omega+\omega^{\prime}\right) / 2}\left[\delta\left(\omega-\omega^{\prime}\right)+\delta\left(\omega+\omega^{\prime}\right)\right] a_{\omega k} a_{\omega^{\prime} k}^{+}+c . c .\right\}, \\
& =\frac{1}{2} \int_{-\infty}^{\infty} \int_{-\infty}^{\infty} \int_{-\infty}^{\infty} d \omega d^{2} k \frac{\omega}{2 \omega \sinh \pi \omega}\left\{a_{\omega k} a_{-\omega-k}+a_{\omega k}^{+} a_{-\omega-k}^{+}\right. \\
& \left.+e^{\pi \omega}\left(a_{\omega k} a_{\omega k}^{+}+a_{\omega k}^{+} a_{\omega k}\right)\right\} .
\end{aligned}
$$

Without much ado one can readily see that the expansion for $H_{I I}$, Eq. (2.8), is nearly the same. Equations (2.15) and (2.16c) dictate the replacement

$$
e^{\pi \omega} \rightarrow e^{-\pi \omega}
$$

Thus $H_{I I}$ decomposes into an analogous mode sum.

$$
\begin{gathered}
H_{I I}=-\frac{1}{2} \int_{-\infty}^{\infty} \int_{-\infty}^{\infty} \int_{-\infty}^{\infty} d \omega d^{2} k \frac{\omega}{2 \sinh \pi \omega}\left\{a_{\omega k} a_{-\omega-k}+a_{\omega k}^{+} a_{-\omega-k}^{+}\right. \\
\left.+e^{-\pi \omega}\left(a_{\omega k} a_{\omega k}^{+}+a_{\omega k}^{+} a_{\omega k}\right)\right\} .
\end{gathered}
$$


4. The Transverse Subsystem Momenta $\overrightarrow{\mathrm{P}}_{\mathrm{I}}$ and $\overrightarrow{\mathrm{P}}_{\mathrm{II}}$.

To express $\vec{P}_{I}$, Eq. (2.11), in terms of the Minkowski Bessel degrees of freedom in Eq. (2.17) is trivial compared to $H_{I}$. The result is, nevertheless, just as interesting. One obtains

$$
\begin{gathered}
\vec{P}_{I}=\frac{1}{2} \int_{-\infty}^{\infty} \int_{-\infty}^{\infty} \int_{-\infty}^{\infty} d \omega d^{2} k \frac{\vec{k}}{2 \sinh \pi \omega}\left\{a_{\omega k} a_{-\omega-k}+a_{\omega k}^{+} a_{-\omega-k}^{+}\right. \\
\left.+e^{\pi \omega}\left(a_{\omega k} a_{\omega k}^{+}+a_{\omega k}^{+} a_{\omega k}\right)\right\} .
\end{gathered}
$$

Similarly for $\vec{P}_{I I}$ in Eq. (2.9) one obtains

$$
\begin{gathered}
\vec{P}_{I I}=-\frac{1}{2} \int_{-\infty}^{\infty} \int_{-\infty}^{\infty} \int_{-\infty}^{\infty} d \omega d^{2} k \frac{\vec{k}}{2 \sinh \pi \omega}\left\{a_{\omega k} a_{-\omega-k}+a_{\omega k}^{+} a_{-\omega-k}^{+}\right. \\
\left.+e^{-\pi \omega}\left(a_{\omega k} a_{\omega k}^{+}+a_{\omega k}^{+} a_{\omega k}\right)\right\} .
\end{gathered}
$$

The K-G subsystem I is characterized by three commuting conserved canonical generators. As one can see from Eqs. (2.20) and (2.22) the Minkowski Bessel modes bring about a nearly complete but not quite total simultaneous diagonalization.

The analogous result for K-G subsystem II is given by Eqs. (2.21) and (2.23).

\section{B. Total System Generators: Complete Diagonalization.}

The generator of boosts for the total Klein-gordon system around $x=t=0$ is merely the sum of the generatos, Eqs. (2.20) and (2.21),

$$
\begin{aligned}
P_{\tau} & =H_{I}+H_{I I} \\
& +\frac{1}{2} \int_{-\infty}^{\infty} \int_{-\infty}^{\infty} \int_{-\infty}^{\infty} \omega\left(a_{\omega k} a_{\omega k}^{+}+a_{\omega k}^{+} a_{\omega k}\right) d \omega d k_{y} d k_{z}
\end{aligned}
$$

Similarly the generator of transverse translations for the total K-G system is the sum of Eqs. (2.22) and (2.23),

$$
\begin{aligned}
\vec{P} & =\vec{P}_{I}+\vec{P}_{I I} \\
& =\frac{1}{2} \int_{-\infty}^{\infty} \int_{-\infty}^{\infty} \int_{-\infty}^{\infty} \vec{k}\left(a_{\omega k} a_{\omega k}^{+}+a_{\omega k}^{+} a_{\omega k}\right) d \omega d k_{y} d k_{z}
\end{aligned}
$$

Thus the Minkowski-Bessel (boost eigenfunction) degrees of freedom bring about a complete diagonalization fo the boost generator and the transverse momentum of the total K-G System.

\section{TRANSLATION GENERATORS FOR THE TOTAL SYSTEM}


An accelerated coordinate frame partitions the total Klein Gordon system into a pair of subsystems. This partitioning is incorporated into the very nature of the Minkowski Bessel modes (by virtue of the event horizons $U=0$ and $V=0$ being the coordinate chart boundaries). Nevertheless these modes are still unitarily related to the Minkowski plane wave modes (see Eq. (2.7). (Physically this means that emission and /or absorption matrix elements computed relative to the plane wave particle quantum state basis are equal to those computed relative to the quantum state basis associated with Minkowski Bessel mode particles.) Symmetry operations and dynamical evolution (in classical variables or quantum states) bring about changes which are generated by the canonical generators. Suppose these generators are expressed in terms of the Minkowski-Bessel degrees of freeedom. Then the acceleration induced partitioning of the total K-G system can manifest itself classically and quantum mechanically through these canonical generators.

Then canonical generator (for the total K-G system) which are of interest are the total inertial energy and momentum, the translation generators,

$$
\begin{aligned}
P_{t} & =\iiint \xi_{t}^{\mu} T_{\mu}^{0} d^{3} x \\
P_{x} & =\iiint \xi_{x}^{\mu} T_{\mu}^{0} d^{3} x \\
P_{y} & =\iiint \xi_{y}^{\mu} T_{\mu}^{0} d^{3} x \\
P_{z} & =\iiint \xi_{z}^{\mu} T_{\mu}^{0} d^{3} x
\end{aligned}
$$

and the total moment of mass energy, the Lorentz boost generator,

$$
P_{\tau}=\iiint \xi_{\tau}^{\mu} T_{\mu}^{\nu} d^{3} \Sigma_{\nu}
$$

The purpose of this subsection is to expand the inertial energy and momentum in terms of the Minkowski Bessel modes. This goal we can achieve in three steps.

(i) Notice that all these generators can be obtained from a single scalar as follows:

Recall the familiar plane we expansion of each generator

$$
P_{\mu}=\frac{1}{2} \iiint k_{\mu}\left(a_{\vec{k}} a_{\vec{k}}^{+}+a_{\vec{k}}^{+} a_{\vec{k}}\right) d^{3} k \quad \mu=0,1,2,3
$$

where $\left(k_{0}, k_{1}, k_{2}, k_{3}\right)=\left(-\omega_{k}, k_{x}, k_{y}, k_{z}\right)$

and $\omega_{k}=\sqrt{k_{x}^{2}+k_{y}^{2}+k_{z}^{2}+m^{2}}>0$ 
Consider the "generating" scalar

$$
\mathcal{P}(x)=\frac{1}{2} \iiint e^{i k_{\mu} x^{\mu}}\left(a_{\vec{k}} a_{\vec{k}}^{+}+a_{\vec{k}}^{+} a_{\vec{k}}\right) d^{3} k .
$$

It follows that

$$
P_{\mu}=\left.\frac{1}{i} \frac{\partial}{\partial x^{\mu}} \mathcal{P}(x)\right|_{x=0} \quad \mu=0,1,2,3
$$

are precisely the four translation generators,

$$
\left(P_{t}, P_{x}, P_{y}, P_{z}\right)=\left(-P_{0}, P_{1}, P_{2}, P_{3}\right) .
$$

Furthermore

$$
\begin{aligned}
P_{\tau} & =-\left.\left[x \frac{\partial}{\partial t}+t \frac{\partial}{\partial x}\right] \mathcal{P}\left(x^{\nu}\right)\right|_{x^{\nu}=0} \\
& =-\left.\frac{\partial}{\partial \tau} \mathcal{P}\left(x^{\nu}\right)\right|_{x^{\nu}=0}
\end{aligned}
$$

Thus all four generators can be obtained by taking appropriate derivatives of the generating scalar $\mathcal{P}(x)$.

(ii) Relabel the modes in terms of the positive mass shell parameters $\theta, k_{y}, k_{z}$, which are defined by

$$
\begin{aligned}
\omega_{k} & =k \cosh \theta ; k_{x}=k \sinh \theta \\
k & =\sqrt{k_{y}^{2}+k_{z}^{2}+m^{2}}
\end{aligned}
$$

and in terms of which the boost (in the $x-t$ plane) invariant measure is

$$
\frac{d^{3} k}{\omega_{k}}=d \theta d k_{y} d k_{z}
$$

In terms of these parameters the Klein-Gordon Minkowski plane wave mode expansion is

$$
\psi(x)=\int_{-\infty}^{\infty} \int_{-\infty}^{\infty} \int_{-\infty}^{\infty}\left(a_{\theta k} f_{\theta k}+a_{\theta k}^{+} f_{\theta k}^{*}\right) d \theta d k_{y} d k_{z},
$$

where

$$
\begin{aligned}
& f_{\theta k} \&=\left[2(2 \pi)^{3}\right]^{-1 / 2} e^{-i\left(\omega_{k} t-k_{x} x\right)} e^{i\left(k_{y} y+k_{z} z\right)} \\
& =\left[2(2 \pi)^{3}\right]^{-1 / 2} e^{-i k\left[(t-x) e^{\theta}+(t+x) e^{-\theta}\right] / 2} e^{\left.i\left(k_{y}(3) \cdot .8\right)^{2} z\right)}
\end{aligned}
$$



by

The familiar dynamical plane wave amplitude $a_{\vec{k}}$ is related to the reparametrized one

$$
a_{\theta k}=\sqrt{k \cosh \theta} a_{\vec{k}}
$$

Insert Eqs. (3.6), (3.8) and (3.9) into (3.3), and the generating scalar becomes

$$
\mathcal{P}(x)=\frac{1}{2} \int_{-\infty}^{\infty} \int_{-\infty}^{\infty} \int_{-\infty}^{\infty} \exp \left[-i k\left(U e^{\theta}+V e^{-\theta}\right) / 2\right] e^{i\left(k_{y} y+k_{z} z\right)}\left(a_{\theta k} a_{\theta k}^{+}+a_{\theta k}^{+} a_{\theta k}\right) d \theta d k_{y} d k_{z}
$$

where $U=t-x ; \quad V=t+x$.

(iii) Implement the unitary transformation $e^{-i \omega \theta} / \sqrt{2 \pi}$. This yields the Minkowski Bessel modes Eq. (2.13) from the relabelled plane wave modes in Eq. (3.8). It also gives the Minkowski Bessel dynamical amplitude

$$
a_{\omega k}=\sqrt{1 / 2 \pi} \int_{-\infty}^{\infty} a_{\theta k} e^{i \omega \theta} d \theta
$$

In terms of these amplitudes and these M-B modes the generating scalar is

$\mathcal{P}(x)=\frac{1}{2} \int_{-\infty}^{\infty} \int_{-\infty}^{\infty} \int_{-\infty}^{\infty} \int_{-\infty}^{\infty} B_{\omega-\omega^{\prime}}^{+}(k U, k V) e^{i\left(k_{y} y+k_{z} z\right)}\left(a_{\omega k} a_{\omega^{\prime} k}^{+}+a_{\omega k}^{+} a_{\omega^{\prime} k}\right) d \omega d \omega^{\prime} d k_{y} d k_{z}$

From this generating scalar one can obtain the total K-G energy and momentum expressed in terms of the M-B degrees of freedom. One must use Eq. (3.4) to do this.

One readily recovers the total transverse momentum, Eq. (2.25), from the generating scalar, Eq. (3.11). Using Eq. (3.4) together with

$$
B_{\omega-\omega^{\prime}}^{ \pm}(0,0)=\delta\left(\omega-\omega^{\prime}\right)
$$

one obtains

$$
\begin{aligned}
\vec{P} & =\left.\vec{\nabla} \mathcal{P}\left(x^{\nu}\right)\right|_{x^{\nu}=0} \\
& =\frac{1}{2} \int_{-\infty}^{\infty} \int_{-\infty}^{\infty} \int_{-\infty}^{\infty} \vec{k}\left(a_{\omega k} a_{\omega k}^{+}+a_{\omega k}^{+} a_{\omega k}\right) d \omega d k_{y} d k_{z} \\
& =\vec{P}_{I}+\vec{P}_{I I}
\end{aligned}
$$

which agrees with Eq. (2.25). 
Similarly one recovers the total moment of mass energy of the field, Eq. (2.24), by inserting (2.16a) into (3.5),

$$
\begin{aligned}
P_{\tau} & =-\left.\frac{\partial}{\partial \tau} \mathcal{P}\left(x^{\nu}\right)\right|_{x^{\nu}=0} \\
& =\frac{1}{2} \int_{-\infty}^{\infty} \int_{-\infty}^{\infty} \int_{-\infty}^{\infty} \omega\left(a_{\omega k} a_{\omega k}^{+}+a_{\omega k}^{+} a_{\omega k}\right) d \omega d k_{y} d k_{z}
\end{aligned}
$$

One can also exhibit the expansions of the longitudital momentum components $P_{t}$ and $P_{x}$ in terms of the Minkowski-Bessel degrees of freedom, but we shall delay doing this until we use them to discuss the longitudinal translational symmetry breaking of the K-G system.

\section{CONCLUSION}

The basic result of this paper is that the total Klein-Gordon system, with its infinite number of dynamical degrees of freedom, is partitioned by an (linearly uniformly) accelerated coordinate frame into a pair of independent dynamical subsystems. This result has been made precise by exhibiting the canonical evolution and symmetry generators for these subsystems (as well as for the total system) in terms of the Minkowski-Bessel degrees of freedom. They are those consitutents of the K-G system which exhibit its partitioning but are still unitarily related to the plane wave degrees of freedom. This Minkowski Bessel mode decomposition of the canonical generators is at the basis of the quantum dynamics

of the acceleration partioned K-G system. These dynamics are discussed elsewhere ${ }^{2}$.

\section{References}

1. U. H. Gerlach, "The Rindler Condensate: Ground State in an Accelerated Frame" in Proceedings of 4th Marcel Grossmann Meeting on General Relativity, R. Ruffini (ed.) (Elsevier Science Publishers B.V., 1986) P1129-1138.

2. U. H. Gerlach, "Liquid Light and Accelerated Frames", Annales de l' I.H.P 49, 397-401 (1988).

3. U. H. Gerlach, "Quantum States of a Field Partitioned by an Accelerated Frame", Phys. Rev. D 40, 1037-1047 (1989)

4. S. A. Fulling, Phys. Rev. D $\underline{7}, 2850$ (1973).

5. W. Israel, Phys. Lett $\underline{57}$ A, 107 (1976).

6. W. G. Unruh, Phys. Rev. D $\underline{14}, 870$ (1976).

7. See, for example, N. J. Vilenkin, Special Functions and the Theory of Group Representations, (American Mathematical Society Providence, R. I., 1968) p.195198, 248-252.

8. U. H. Gerlach, "Minkowski Bessel Modes", Phys. Rev. D 38, 514-521 (1988); gr-qc/9910097. 